\title{
Fast Photochemistry of Prototypical Phytochromes-A Species vs. Subunit Specific Comparison
}

\author{
Janne A. Ihalainen ${ }^{1 *}$, Heikki Takala ${ }^{1,2}$ and Heli Lehtivuori ${ }^{1,3}$ \\ ${ }^{1}$ Department of Biological and Environmental Sciences, Nanoscience Center, University of Jyväskylä, Jyväskylä, Finland, \\ ${ }^{2}$ Department of Anatomy, Institute of Biomedicine, University of Helsinki, Helsinki, Finland, ${ }^{3}$ Department of Physics, \\ Nanoscience Center, University of Jyväskylä, Jyväskylä, Finland
}

OPEN ACCESS

Edited by:

Tilo Mathes,

Vrije Universiteit Amsterdam

Netherlands

Reviewed by:

Derren Heyes,

University of Manchester, UK

Rolf Diller,

University Kaiserslautern, Germany

*Correspondence:

Janne A. Ihalainen

janne.ihalainen@jyu.fi

Specialty section:

This article was submitted to Biophysics,

a section of the journal

Frontiers in Molecular Biosciences

Received: 08 August 2015 Accepted: 07 December 2015 Published: 23 December 2015

Citation:

Ihalainen JA, Takala H and Lehtivuori H (2015) Fast Photochemistry of Prototypical Phytochromes -A

Species vs. Subunit Specific Comparison. Front. Mol. Biosci. 2:75. doi: 10.3389/fmolb.2015.00075
Phytochromes are multi-domain red light photosensor proteins, which convert red light photons to biological activity utilizing the multitude of structural and chemical reactions. The steady increase in structural information obtained from various bacteriophytochromes has increased understanding about the functional mechanism of the photochemical processes of the phytochromes. Furthermore, a number of spectroscopic studies have revealed kinetic information about the light-induced reactions. The spectroscopic changes are, however, challenging to connect with the structural changes of the chromophore and the protein environment, as the excited state properties of the chromophores are very sensitive to the small structural and chemical changes of their environment. In this article, we concentrate on the results of ultra-fast spectroscopic experiments which reveal information about the important initial steps of the photoreactions of the phytochromes. We survey the excited state properties obtained during the last few decades. The differences in kinetics between different research laboratories are traditionally related to the differences of the studied species. However, we notice that the variation in the excited state properties depends on the subunit composition of the protein as well. This observation illustrates a feedback mechanism from the other domains to the chromophore. We propose that two feedback routes exist in phytochromes between the chromophore and the remotely located effector domain. The well-known connection between the subunits is the so-called tongue region, which changes its secondary structure while changing the light-activated state of the system. The other feedback route which we suggest is less obvious, it is made up of several water molecules ranging from the dimer interface to the vicinity of the chromophore, allowing even proton transfer reactions nearby the chromophore.

Keywords: red photosensors, excited state dynamics, fluorescence, transient absorption, laser spectroscopy

\section{INTRODUCTION}

Phytochromes are red light-sensing photosensory proteins that exist in plants, fungi, and bacteria. The incident light leads to several structural and chemical changes of the protein, and thus, controls its biological activity. The structural changes between the two (thermodynamically stable) light-switchable states are considerably large in the photosensory module of the bacteriophytochromes (Takala et al., 2014a). The far-red fluorescence emission 
properties of phytochromes offer potential to tissue imaging (Fischer and Lagarias, 2004). Due to relatively low scattering, lower light absorption in living tissue, and good tissue penetration, the red light-sensing proteins provide an advantage over other photosensory proteins. The potential of phytochromebased optogenetic switches have already been recognized by several laboratories (Shimizu-Sato et al., 2002; Möglich and Moffat, 2010; Piatkevich et al., 2013a,b; Gasser et al., 2014).

Phytochromes are widely found in the bacterial kingdom. A comprehensive description of various species, their occurrence, and function, is represented elsewhere (for example the review of Auldridge and Forest, 2011). On the other hand, the time-resolved spectroscopic studies of phytochromes have concentrated on a rather small set of phytochromes. We focus on phytochrome species which contain canonical domain architecture (Figure 1). We also concentrate on phytochromes whose light-activated reactions from $\mathrm{Pr}$ to Lumi-R have been studied on the ultra-fast time scales. These are the phytochromes from Agrobacterium tumenfaciens (A. tumenfaciens, Agp1), Synechocystis sp. PCC 6803 (cyanobacterial phytochrome, Cph1), Deinococcus radiodurans (D. radiodurans, DrBphP), Rhodopseudomonas palustris (R. palustris, RpBphP2 and $R p B p h P 3)$, and Stigmatella aurantiaca, SaBphP1. The chromophore of the bacteriophytochrome is an open tetrapyrrole bilin molecule (Figure 1). In the case of Cph1, the chromophore is phycocyanobilin (PCB), The PCB differs from the BV by the lack of double bond character in the A ring and an ethyl-group in the $\mathrm{C} 18$ position. The plant phytochromes carry either $\mathrm{PCB}$ or phytochromobilin (РФB) (Rockwell et al., 2006).

\section{PROTEIN CONSTITUENTS AND THE PHOTOACTIVE STATES}

A canonical bacteriophytochrome functions as a homodimer and consists of four different protein domains (Figure 1). The photosensory unit is made up of so-called PAS (PER, ARNT, SIM), GAF (cGMP phosphodiesterase, adenylate cyclase, FhlA), and PHY (Phytochrome-specific GAF related) domains. The PAS and GAF domains are together called a chromophore-binding domain (CBD). In prokaryotes, the bilin-binding residue resides in the PAS domain, whereas in cyanobacteria and plants the PCB and $\mathrm{P} Ф \mathrm{~B}$ pigments are ligated with the GAF domain (Wagner et al., 2007). The fourth subunit, C-terminal of the PHY-domain, functions as biological effector and is often histidine kinase domain (HK). In addition to this canonical domain composition, a large set variation in the domain architecture exists in different phytochrome types. For example, Synechocystis Cph2 lacks the PAS domain, while cyanobacteriochromes lack both PAS and PHY domains and function as multi-subunit GAF domains (Rockwell et al., 2006).

The two photostable states of phytochromes are called Pr (red-absorbing state) and Pfr (far-red-absorbing state). The phytochromes with the $\operatorname{Pr}$ as dark resting state, like Agp1, Cph1, DrBphP, RpBphP2, and $\mathrm{SaBphP1}$, are called prototypical phytochromes. The bacteriophytochromes, like Agp2 from $A$. tumenfaciens and a phytochrome from Pseudomonas aeruginosa
( $P$. aeruginosa, $\mathrm{PaBphP}$ ) thermally revert to the $\mathrm{Pfr}$ state and are called bathy phytochromes. In plants where a large variety of phytochrome isoforms exist, most of the phytochromes are prototypical.

The recent structural information has lifted the understanding about the phytochrome function considerably (Vierstra and Zhang, 2011; Burgie and Vierstra, 2014). The CBD fragment of DrBphP was the first ever-published phytochrome structure in atomic resolution (Wagner et al., 2005, 2007). This structure confirmed the bilin-binding pocket and the conformation of BV as a $Z Z Z Z_{\text {ssa }}$ conformation (Figure 1) (Wagner et al., 2005). It revealed a peculiar figure-of-eight-knot structure which bridges the PAS and GAF domains. The refined CBD structure confirmed how $\mathrm{C}^{2}$ in the vinyl group in the A-ring of the BV binds via a thioether linkage to the protein. Higher resolution structures revealed a number of coordinated water molecules and buried contacts between the monomeric units as dimerization sites (Wagner et al., 2007). Later, Auldridge et al. utilized this information for the production of monomeric CBD protein (Auldridge et al., 2012). Comparison with the CBD structures of other species set an important basis in the understanding about the photoconversion mechanism of the bilin molecules in the binding pocket (Yang et al., 2007). The high-resolution structures of CBD proteins have naturally been highly beneficial in the design of phytochrome-based near-infrared fluorescent proteins (Shu et al., 2009; Filonov et al., 2011; Auldridge et al., 2012; Shcherbakova and Verkhusha, 2013; Bhattacharya et al., 2014; Yu et al., 2014).

The structures of the full photosensory module (CBDPHY) of Cph1 (Essen et al., 2008), PaBphP (Yang et al., 2008, 2009), DrBhP (Burgie et al., 2014a; Takala et al., 2014a), and a PhyB isoform from Arabidopsis thaliana (Burgie et al., 2014b) have been reported. The structure of the photosensory module resembles a tandem-GAF arrangement with a long connecting helix backbone (Essen et al., 2008). The PHY domain extends near to the chromophore by a so-called tongue-region which has a $\beta$-hairpin structure or an $\alpha$-helical structure in the prototypical and bathy phytochromes, respectively, in their resting state (Essen et al., 2008; Yang et al., 2008). This tongue region contains a conserved $\mathrm{PRxSF}$ motif that interacts with the GAF domain near the chromophore and blocks the solvent accessibility to the chromophore-binding pocket. In the $\mathrm{Pr}$ state, this tongue motif forms a salt bridge between residues Asp207 and Arg466. The structural studies confirmed the15Za and the 15Ea conformations of the biliverdin in the Pr state and $\mathrm{Pfr}$ state, respectively. The $\mathrm{BV}$ isomerization leads to changes in the PHY-GAF interaction matrix. The $\beta$-hairpin structure in the tongue region disappears and an $\alpha$-helical structure is stabilized. In the same process, a separation of the sister PHY domains was observed (Takala et al., 2014a). At the moment, the high-resolution structural information of the full-length phytochrome is missing and we need to settle for electron microscopic information (Burgie et al., 2014a,b). Solidstate magic-angle spinning NMR spectroscopy has also revealed detailed information about the hydrogen bond network around the chromophore (Song et al., 2011). Most of the studies have been conducted with Cph1 and oat PhyA proteins, however. 


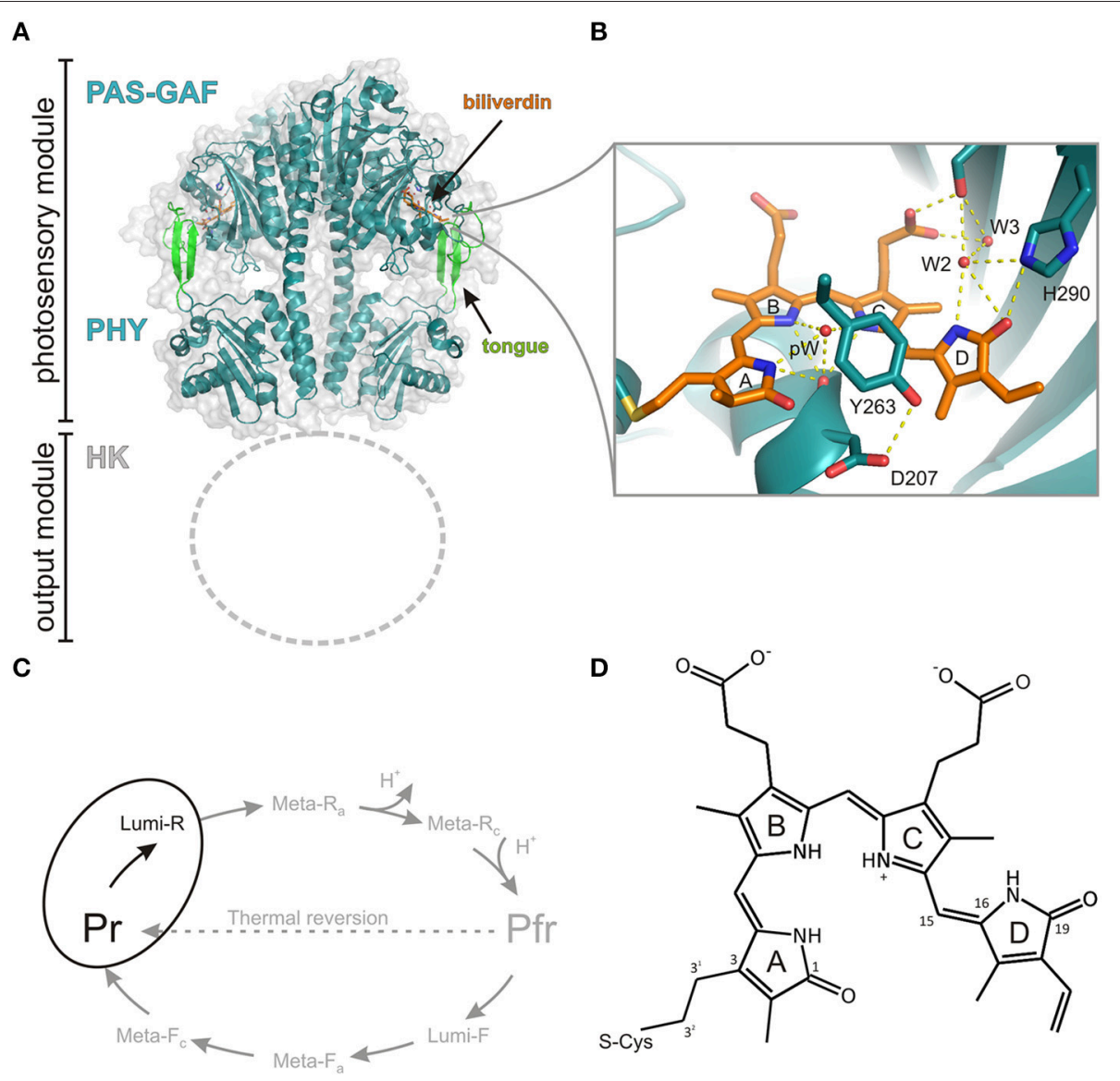

FIGURE 1 | Structure and photocycle of a canonical phytochrome from Deinococcus radiodurans. (A) The photosensory module of the phytochrome (PDB code 4OOP, Takala et al., 2014a) forms a parallel dimer that consists of chromophore-binding PAS and GAF domains, which are followed by a PHY domain. Due to the lack of structural information, the N-terminal histidine kinase (HK) domain is not shown. (B) A closed view of the biliverdin chromophore and its selected interactions to three water molecules pW (pyrrole water), W2, W3 and amino acids (Asp207, Tyr263, and His290). The panel is based on the high-resolution structure of the CBD fragment (PDB code 4QOH, Burgie et al., 2014b). (C) Photocycle of the phytochrome with its intermediates. In this study, we concentrate on the first step of the forward reaction ( $\mathrm{Pr}->$ Lumi-R), highlighted in black. (D) The structure of the biliverdin molecule. The key atoms are numbered.

The kinetic information between the Pr and Pfr states relies on visible and vibrational spectroscopic results. The spectroscopic results are, however, difficult to link directly with the structural and chemical changes of the protein. The transition between $\mathrm{Pr}$ and Pfr state contains intermediate states (Figure 1), initially determined by UV-Vis absorption spectral changes at various temperatures (Eilfeld and Rüdiger, 1985). A similar method has also been used for the characterization of these states by the means of FTIR-spectroscopy (Foerstendorf et al., 2001; Schwinté et al., 2009; Piworski et al., 2010) and FT-Raman spectroscopy (Matysik et al., 1995). Due to the resonance-Raman conditions the signal assignment of the Raman spectra concentrates on the bilin vibrational modes. The FTIR-spectroscopy reveals information also from the protein and the assignment of IRabsorption spectrum is more challenging (Foerstendorf et al., 2001; Barth and Zscherp, 2002; Schwinté et al., 2009; Piworski et al., 2010; Stojkovicì et al., 2015; Velazquez Escobar et al., 2015). The clearest changes are in the $1730 \mathrm{~cm}^{-1}$ region, which reports the carbonyl vibrations of the chromophore. Several
Amide I transitions have been indicated to the changes in the secondary structure of the protein during the reaction. The first intermediate, which is formed from the excited state bilin molecule is called Lumi-R state (Figure 1). It has a characteristic, slightly red-shifted absorption band. The transition between excited $\mathrm{Pr}^{*}$ to Lumi-R takes place in ps-ns range as it occurs via the excited state of the bilin molecule. The Pr to Lumi-R reaction is the gateway reaction to the photocycle (Figure 1). The quantum yield of the total Pr to Pfr photo reaction is mainly determined by the Pr to Lumi-R-reaction although a back reaction channel from Lumi-R to Pr state has been observed with a time-scale of 100 ns (Mathes et al., 2015). Typically, the fast photo processes are studied by means of ultrafast transient absorption techniques, either in the visible region or in the midinfrared region, but also fluorescence techniques have been used for determining the excited state lifetimes. The description of this transition will come later in the ultra-fast spectroscopy section. The Lumi-R state transfers to so-called Meta- $\mathrm{R}_{a}$ state in about $100 \mu$ s time scale and shows a further red-shifted absorption. The 
next transition is Meta- $\mathrm{R}_{\mathrm{a}}$ to Meta- $\mathrm{R}_{\mathrm{c}}$ transition and it takes place in $\mathrm{ms}$ time scale, after which the protein undergoes the Meta$\mathrm{R}_{\mathrm{c}}$ to Pfr reaction. During these phases, kinetic proton transfer reactions take place (van Thor et al., 2001; Borucki et al., 2005). In the transition from Meta- $R_{a}$ to Meta- $R_{c}$ state a proton is released to the solvent which is again taken up by the protein in the Meta$\mathrm{Rc}$ to Pfr reaction. The spectroscopic character during these reactions is a decrease of the extinction coefficient at most of the spectral region and a final far-red shift of the absorption. Thus, the decrease of the absorption intensity represents the proton release mechanism in the protein. The site(s) of the released and reclaiming site(s) of protons are unknown, however. A recent study suggests a model of the proton transfer pathway and a tautomeric system in bathy phytochromes (Velazquez Escobar et al., 2015), initially suggested by Lagarias and Rapoport (1980).

Probably due to crystal packing effects, the studies of intermediate states with crystallography-based techniques have been challenging. Up to present, the nature of the various intermediate states has been studied structurally by the means of cryotrapping X-ray crystallography (Yang et al., 2011). Detailed structural changes in the chromophore-binding pocket under illumination at the temperature range of $-180^{\circ} \mathrm{C}$ to $-120^{\circ} \mathrm{C}$ report the initial changes of the chromophore. Besides temperature-dependent experiments, rather extensive mutagenesis approaches have been linked to resonance Raman experiments. Several site-selective mutations in the vicinity of the chromophore (like in Asp207, Tyr263, His290, see Figure 1) or in the tongue region (e.g., Arg466) block the photocycle to a certain intermediate state, which can then be then probed by resonance Raman spectroscopy (Wagner et al., 2008).

\section{ULTRA-FAST KINETICS OF THE PR* TO LUMi-R -TRANSITION}

Plant phytochromes were the first phytochrome systems to be studied with ultra-fast spectroscopic methods (Sineshchekov, 1995). The initial photoprocesses of the oat phytochrome were determined to be around $30 \mathrm{ps}$. Similar photoactivated reaction times have been determined for cyanobacterial Cph1 (Heyne et al., 2002; van Thor et al., 2007; Kim et al., 2013). The timeresolved IR-spectroscopy (tr-IR) follows the most intimately of the structural changes of the chromophore and/or its protein environment in the $\mathrm{Pr}^{*}$ to Lumi-R reaction. Recently, by using polarized tr-IR experiments (Yang et al., 2012, 2014) elegantly recorded the orientation of $\mathrm{C}_{19}=\mathrm{O}$ bond of the $\mathrm{D}$-ring after photoexcitation and thus demonstrated the action of the $\mathrm{Pr}^{*}$ to Lumi-R reaction. The time constant for the rotation of the D-ring was reported being about $30 \mathrm{ps}$ in Cph1 $\Delta 2$ (CBD-PHY). In addition, two different PCB orientations were detected in the resting $\operatorname{Pr}$ state with significantly different $\mathrm{H}$ bond networks and different rotation yields for both starting orientations (Yang et al., 2014). Longer reaction lifetimes have been reported for bacteriophytochromes, where the excited-state reactions were slower, about 100-300 ps (Toh et al., 2011a,b; Lehtivuori et al., 2013; Mathes et al., 2015). The Agp1 shows a 30 ps photoproduct formation (Schumann et al., 2007; Linke et al., 2013), which would indicate more similar lifetimes with the plant and cyanobacterial phytochromes. However, the lifetime results from $\mathrm{DrBphP}, \mathrm{PaBphP}$, and $\mathrm{SaBphP}$, are from truncated systems. By plotting the kinetics of the full-length system with the truncated constructs in (Figure 2), we show clearly longer decay times in the transient absorption data and fluorescence data of the shorter constructs than in the full-length system, in line with (Toh et al., 2011a,b; Lehtivuori et al., 2013; Mathes et al., 2015).

In ultra-fast spectroscopic studies, it has become clear that the excited state decay is complex with multi-exponential kinetics (Sineshchekov, 1995). The multi-exponential decay profiles indicate the multiple pathways of the excited Pr* state, including sub-ps S1-relaxation processes, fluorescence, and (multiple) nonradiative (productive and non-productive) decay channels. By using two different excitation wavelengths and a rate distribution modeling, Heyne et al. observed a different type of excited-state kinetics for Cph1 (Heyne et al., 2002). Multi-pulse experiments in the transient absorption data have provided interesting details on the excited-state dynamics of the Pr* states (Kim et al., 2013, 2014). In these experiments a "fluorescing" pool, non-radiative decay pool, and a reactive pathway with the time constants of the reactions between each of the pools are identified (Kim et al., 2014).

All of the above-mentioned studies indicate that the phytochrome systems contain strong non-productive channels. This has a consequence that the photochemical yield of the $\mathrm{Pr}^{*}$ to Lumi-R transition is low. In all studied species it has been shown to be between 0.1 and 0.2 for cyanobacterial phytochromes (Schumann et al., 2007; van Thor et al., 2007) and 0.05-0.15 for bacteriophytochromes (Toh et al., 2010; Mathes et al., 2015; Lehtivuori et al., unpublished).

In addition to the multiple decay pathways, the multiexponential decay profile of the phytochromes may indicate the heterogeneity of the system. The heterogeneity vs. the homogeneity of the Pr state has been under debate the last decade. With NMR-studies (which probes solely the electronic ground states), Song et al. (2011) stated the presence of multiple Pr states in the Cph1 system, whereas the Pfr state is homogenous. Also low temperature single-molecule spectroscopic and site-specific fluorescence experiments indicate heterogeneity in the Pr state in several species (Nieder et al., 2009, 2013; Sineshchekov et al., 2014; Yang et al., 2014). In fact, Nieder et al. revealed, in addition to the heterogeneity between individual particles, spectral diffusion among single particles (Nieder et al., 2009). Thus, the phytochromes switch between the spectral forms even at very low temperatures. Room temperature Raman experiments with the Cph1 systems demonstrate the homogenous behavior of the absorption profile (Dasgupta et al., 2009; Spillane et al., 2009).

\section{EXCITED STATE LIFETIMES-TIME-RESOLVED FLUORESCENCE STUDIES}

As mentioned above, a fraction of the excitation in the phytochrome system is emitted as fluorescence and 

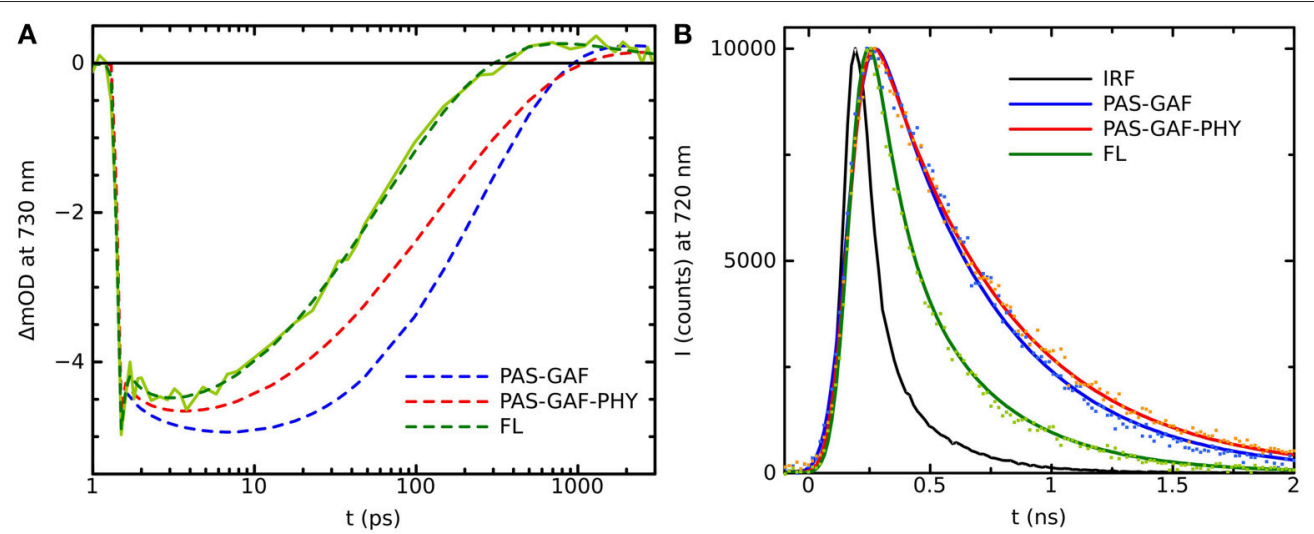

FIGURE 2 | (A) Transition absorption decay traces of PAS-GAF (blue), PAS-GAF-PHY (red), and FL (green) from Deinococcus radiodurans excited at $656 \mathrm{~nm}$ and monitored at $730 \mathrm{~nm}$. Solid lines show the multiexponential fit of the data, which result in following characteristic lifetimes of each complexes are: 1.5 , ps and $320 \mathrm{ps}$ for CBD; 1.2 ps and 170 ps for CBD-PHY; 0.5 ps and 70 ps for FL. (B) Emission decays of PAS-GAF, PAS-GAF-PHY, and FL from Deinococcus radiodurans excited at $660 \mathrm{~nm}$ and monitored at $720 \mathrm{~nm}$. IRF is the instrument response function. The corresponding average lifetimes are: 410 ps for CBD; 550 ps for CBD-PHY; 340 ps for FL. The fluorescence quantum yields are $0.034,0.017$, and 0.008 for CBD, CBD-PHY, and FL, respectively. The samples were prepared as described in Takala et al. (2014b). The pump-probe technique for time-resolved absorption was used to detect fast processes with a time resolution shorter than $0.2 \mathrm{ps}$. A laser setup with an integrated one-box femtosecond Ti:sapphire laser (Quantronix Integra C) was used to pump two home-built non-collinear optical parametric amplifiers (NOPAs) to produce the $(656 \pm 14) \mathrm{nm}$ pump pulses. A white light continuum generated in a sapphire crystal was used for probing. After the sample, the probe and reference beams entered a monochromator (Acton), and the detection was set to (730 \pm 8$) \mathrm{nm}$. Fluorescence decays of the samples in the nanosecond time scales were measured using a time-correlated single photon counting (TCSPC) system (PicoQuant GmBH). The excitation wavelengths were $660 \mathrm{~nm}$. The monochromator (Jobin Yvon) was used to detect the emission at $720 \mathrm{~nm}$ with a single photon avalanche photodiode (MPD). To avoid excessive sample degradation in both time-resolved measurements, the sample solution was cycled using a peristaltic pump (Ismatec). A far-red diode at (750 \pm 5) nm (Leading-Tech Laser Co.) was used to transform the sample to the Pr state by constantly illuminating. More details in Lehtivuori et al. (2013).

phytochromes offer great potential for far-red fluorescent proteins (Fischer and Lagarias, 2004; Miller et al., 2006; Shu et al., 2009; Filonov et al., 2011; Auldridge et al., 2012; Shcherbakova and Verkhusha, 2013; Bhattacharya et al., 2014; Yu et al., 2014; Shcherbakova et al., 2015). However, regardless of the low photochemical yield, wild-type phytochromes are typically poorly fluorescent with fluorescence yields ranging from 0.01 to 0.04 (Fischer and Lagarias, 2004; Toh et al., 2010; Zienicke et al., 2011; Auldridge et al., 2012). Often, the low fluorescence yield is linked to photoisomerization activity (i.e., $\operatorname{Pr}^{*}$ to Lumi-R production). This, however, is a misconception as the largest decay channel for phytochromes systems are typically the nonproductive channels (neither fluorescent nor Lumi-R-forming channel). This can be rationalized from the results of the fluorescence lifetime experiments. As stated above, the initial photoreaction of the cyanobacterial Cph1 take place in about $30 \mathrm{ps}$, but their fluorescence lifetimes have been measured to be around $1 \mathrm{~ns}$ (Otto et al., 2003; Miller et al., 2006). The same is true for plant systems (Sineshchekov, 1995). In bacteriophytochromes the difference between transient absorption and the transient fluorescence experiments is smaller (Figure 2) although in the case of Agp1 the photochemical reaction, from $\mathrm{Pr}^{*}$ to Lumi-R, is also around 30 ps (Schumann et al., 2007; Linke et al., 2013). The photoreaction times and fluorescence lifetimes of various species are gathered in the Table 1. At first glance, the difference between the reported photoreaction times and fluorescence lifetime, together with the low quantum yields, appear puzzling. In fact, fluoroproteins with excited-state lifetimes of about 2 ns and switching ability, would be good fluorescent proteins. For example, the excited state decay times of GFP are 2.8 and
3.3 ns (Striker et al., 1999) and the fluorescence yield can be as high as 0.8 . In principle, the time-resolved fluorescence reflects the general lifetime of the excited state and reveals information about the initial photochemistry. There is, however, a caveat. By using ps laser pulses, which are typically used in single-photon counting set ups, only processes slower than about 100 ps are recorded. As fluorescence rates are considerably slower than the photochemical reactions of the phytochromes, only the fluorescence process is detected and other photo-activated processes remain underneath of the excitation pulse. In addition, other fluorescent channels, like the Lumi-R state (Sineshchekov, 1995), may influence to the lifetime experiments as the mixtures of Lumi-R and Pr fluorescent states are detected.

\section{DISCUSSION}

We have summarized the key observations of the excited state reactions of the phytochrome systems. In the case of plant and cyanobacterial systems the excited-state reactions take place in about 30 ps and the fluorescence lifetime is above 1 ns. If the photoisomerization is impaired, the excited-state lifetimes can be increased up to $3.2 \mathrm{~ns}$. In the bacteriophytochrome systems the photoreaction and excited-state decay processes have similar photoreaction excited state lifetimes, between 100 and $300 \mathrm{ps}$, and with site-selective mutations the lifetime can be increased to $870 \mathrm{ps}$ (Bhattacharya et al., 2014). As the plant and cyanobacterial systems bind $\mathrm{PCB}$ and РФВ chromophores and the bacteriophytochromes BV chromophore, it is clear that the type of the pigment has a role in the excited state lifetime. 
TABLE 1 | Excited state lifetimes of phytochrome systems from various species.

\begin{tabular}{|c|c|c|c|c|c|}
\hline Species & Construct & Cof & Pr lifetime & Yield (\%) & References \\
\hline Agp1 & FL & BV & 25 ps (ppf), $540 \mathrm{ps}^{\star}(\mathrm{flt})$ & 9 & Schumann et al., 2007; Linke et al., 2013 \\
\hline DrBphP & PAS-GAF & BV & 300 ps, 410 (flt) & & Lehtivuori et al., 2013; Figure 2 \\
\hline DrBphP & PAS-GAF-PHY & BV & 170 ps, 550 (flt) & & Figure 2 \\
\hline DrBphP & FL & BV & 70 ps, 340 (flt) & & Figure 2 \\
\hline RpBphP2 & PAS-GAF & BV & 175 ps & & Toh et al., 2011a,b \\
\hline RpBphP2 & PAS-GAF-PHY & BV & $58 \mathrm{ps}$ & 13 & Toh et al., 2011a,b \\
\hline RpBphP3 & PAS-GAF & BV & 300 ps & & Toh et al., 2011a,b \\
\hline RpBphP3 & PAS-GAF-PHY & BV & 330 ps & & Toh et al., 2011a,b \\
\hline SaBphP1 & PAS-GAF & BV & 225 ps & & Mathes et al., 2015 \\
\hline SaBphP1 & PAS-GAF-PHY & BV & $85 \mathrm{ps}$ & & Mathes et al., 2015 \\
\hline Cph1 & PAS-GAF & PCB & 30 ps (ppf) & 15 & Heyne et al., 2002 \\
\hline Cph1 & PAS-GAF-PHY & PCB & 25 ps (ppf) & 13 & Heyne et al., 2002; Yang et al., 2014 \\
\hline Cph1 & FL & PCB & 60 ps (ppf), 1.2 ns (flt) & & Otto et al., 2003; Kim et al., 2014 \\
\hline Cph1 & FL & PEB & $\mathrm{ND}(\mathrm{ppf}) 3.2 \mathrm{~ns}$ (flt) & & Otto et al., 2003 \\
\hline PhyB, Oat & $\mathrm{FL}$ & PCB & $24 \mathrm{ps}$ & 15 & Andel et al., 1997 \\
\hline PhyA, Oat & $\mathrm{FL}$ & PCB & $24 \mathrm{ps}$ & & Müller et al., 2008 \\
\hline PhyA, Oat & $65 \mathrm{kDa}$ & PCB & 24 ps & & Müller et al., 2008 \\
\hline
\end{tabular}

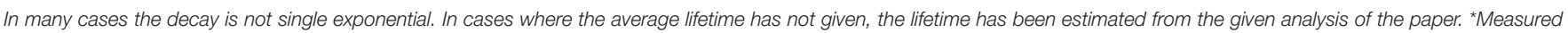
with locked BV, ppf, photoproduct formation; flt, fluorescence lifetime; Cof, Cofactor.

We emphasize, however, that the excited state reactions of the phytochromes are complex. Three processes, photoisomerization, fluorescence, and non-photochemical quenching, are competitive, and we still lack a comprehensive picture of these reactions. One of the main stumbling blocks is the description of the interaction lattice of the bilin molecule with its environment. In the highest resolution structural models, obtained from the CBD systems (Wagner et al., 2007; Auldridge et al., 2012; Burgie et al., 2014b) the amino acid positions as well as the oxygen atoms of the water molecules are well-described. Different species show a different amino acid arrangement in the chromophore binding pocket (Mathes et al., 2015). It is, however, too straightforward to link an effect of single amino acid change in the structure directly to the excited state reaction, such as isomerization process. For example, the protonation states of the chromophore and its nearby histidine-residues influence the photochemical behavior of the molecule. Moreover, a labile protonation state can lead to several different conformations of the amino acids around the chromophore, and thus, heterogeneity in the system. Such effects are invisible in the X-ray crystal structures of the protein complexes.

The great sensitivity of the excited state behavior of the chromophore makes possible for other subunits to influence the photochemical reactions of the chromophore from the larger distances. We have shown that on top of the variation in photo-excitation kinetics among different phytochrome species, each type of construct, i.e., the chromophore-binding domain (CBD), the photosensory core (CBD-PHY), or the full-length phytochrome, show differences in the excited state kinetics. We interpret this variation as the feedback mechanisms of the $\mathrm{PHY}$ and effector (HK) domains to the CBD domain. The first feedback route is the tongue of the PHY domain (Figure 1). In the Pr state, a salt bridge has formed between the Asp207 (from CBD) and Arg466 (in the PHY tongue) whereas in Pfr state the Asp207 coordinates with Ser468 of the tongue (Takala et al., 2014a). The Asp207, part of the conserved DIP motif, locates in the central position in the chromophore-binding pocket and locks the so-called pyrrole (pW in Figure 1B) water in its place. Furthermore, the interactions between the sister HK domains may stabilize the PHY domain orientation and further stabilize the chromophore binding pocket via tongue interactions. Moreover, the tongue controls solvent access to the chromophore-binding pocket. Thus, in the case of truncated CBD systems more water molecules occupy the chromophore binding pocket than in the CBD-PHY and FL-systems. The second feedback route could be a trail of water molecules from the protein interior to the nearby $\mathrm{D}$-ring of the chromophore. In our opinion, the water lattices from the protein interior to the nearby chromophore have gained too little attention. The water molecule(s) nearby the $\mathrm{NH}$-group and the $\mathrm{CO}$ group of the D-ring, marked as W2 and W3 in (Figure 1) and (Figure 3) certainly have $\mathrm{H}$-bond character to the $\mathrm{D}$-ring in the Pr-state and play role in the reaction to Lumi-R. These water molecules seem to be rather conserved in the structures of each species published to date and allow (water mediated) hydrogen-bonding network from deeper sites of the protein scaffold, and possibly, proton transfer pathways as well. Unfortunately, it is very demanding to perform a systematic study about the effect of the H-bond network of these water molecules. We propose that these differences are enough to build up slightly different microenvironment around the pigment in its ground and excited state so that it influences the excitation state kinetics of the systems. 


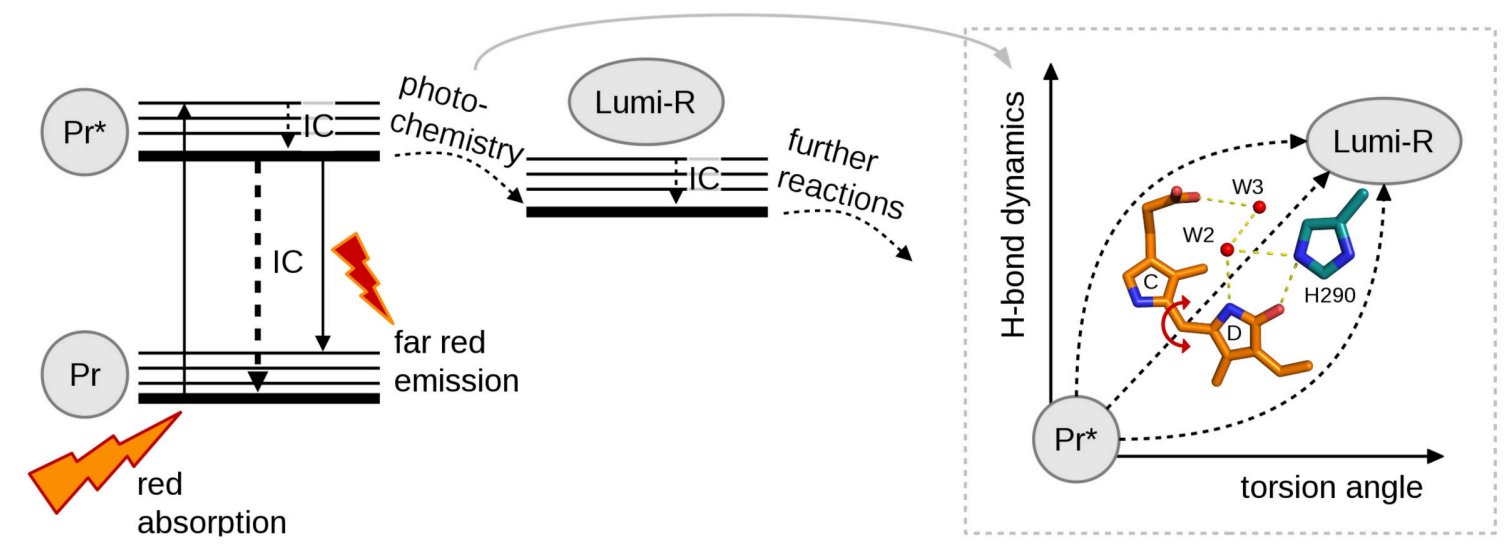

FIGURE 3 | A simplified reaction diagram showing the excitation of molecule with red light from Pr ground state to its singlet excited state (Pr*) followed by internal conversion (IC), fluorescence or photochemistry. Pr relaxes back to the ground state by IC or far-red fluorescence. For photochemistry at least two coordinates are needed to describe the complete reaction, torsion angle and $\mathrm{H}$-bond dynamics.

\section{THE REACTION COORDINATES TOWARD PRODUCTIVE LUMI-R STATE}

Full understanding about the photochemical reaction of phytochromes requires to reveal the most representative reaction coordinate along which the system proceeds from excited $\mathrm{Pr}^{*}$ state to Lumi-R state. Figure 3 summarizes the two main coordinates involved in reaction and how they are linked to structural changes. Temperature-dependent spectroscopic experiments have revealed a barrier along a reaction coordinate, with the activation energy of about $5 \mathrm{~kJ} / \mathrm{mol}$ (Sineshchekov, 1995; Andel et al., 1996; Kim et al., 2013), which corresponds for example to the strength of one hydrogen bond in a system. By using temperature-dependent fluorescence measurements, a small barrier $(2-3 \mathrm{~kJ} / \mathrm{mol})$ has been determined in a so-called Pre-Lumi-R to Lumi-R step in Cph1 (Sineshchekov et al., 2014). An obvious reaction coordinate would be the torsional rotation of the D-ring of the bilin chromophore. Rockwell et al. (2009) demonstrated by using circular dichroism spectroscopy that the $\mathrm{C} 15=\mathrm{C} 16$ isomerization, or the rotation of the D-ring, occurs clockwise in the biliverdin phytochromes whereas the rotation is counter-clockwise in the phytobilin phytochromes (Rockwell et al., 2009). Just following the reaction coordinate of "torsion angle," however, is not sufficient for describing the complete reaction. The other coordinate, called "H-bond dynamics" in (Figure 3), has actually many dimensions. The hydrogen bond network can be described between the D-ring and several amino acids and water molecules in its vicinity (Figure 3). Actually, rather similar amino acid composition around the Dring (His290 in case of D. radiodurans and in Cph1, with an additional H-bond network of Lys183 and Ser297 in case of $R p B p h P 3$, and diminished $\mathrm{H}$-bonding character in the case of $\mathrm{SaBphP1)}$ has been reported. Still, these species show different excited-state lifetimes (Table 1).

The non-productive channels of excitation energy are very dominant in all phytochrome systems and they are challenging to describe. Kennis and co-workers have put forward one potential pathway for excited state decay, namely an excited-state proton transfer reaction, which is suggested to take place among the pyrrole nitrogens of the chromophore, the pyrrole waters and their coordinating amino acid, Asp207 (Toh et al., 2010; Nieder et al., 2013). Other non-productive channels are most likely related to the tumbling of the D-ring, as its stabilization of the D-ring by the hydrogen bond network leads to stronger fluorescent molecules.

To increase the quantum yield of the fluorescence, internal conversion and photochemistry channels are to be diminished, either by protein mutations or by inserting chromophores with impaired photoisomerization capability (Shcherbakova et al., 2015). By using site-selective mutations for the stabilization of the chromophore D-ring environment has been shown to lead higher fluorescence quantum yields in bacteriophytochromes (Shu et al., 2009; Auldridge et al., 2012; Bhattacharya et al., 2014; Yu et al., 2014). An additional increase in the fluorescence yield may be obtained by rigidifying of the protein scaffold part (Bhattacharya et al., 2014). The photoisomerization pathway can be blocked by incorporating the apoprotein with phycoerythrobilin, PEB, which lack the double bond at the $\mathrm{C} 15=\mathrm{C} 16$ position of the chromophore (Figure 1). In this case, the strain for isomerization is lost and excitation does not lead to the isomerization process. Another way of blocking the isomerization process is to use a BV15Za chromophore where $\mathrm{C}$ and D-rings are bridged with an additional linker preventing the rotation of the D-ring (Inomata et al., 2005). In these cases, stronger fluorescence with longer excited state lifetimes, up to $3.2 \mathrm{~ns}$, have been reported for cyanobacterial and bacterial phytochromes (Heyne et al., 2002; Otto et al., 2003; Miller et al., 2006; Zienicke et al., 2011; Kim et al., 2014).

Finally, we would like to point out that the low quantum yields of the photoproductive states are critical only in the 
situations where low flux, or ultra-fast femtosecond pulses need to be used. For the studies of slower, thermally driven reactions, ns-laser pulses with sufficient excitation fluxes can be used. As the spectral shift of phytochrome is so large due to the light activated reaction, multiple excitation lead to full photoconversion of the protein ensemble. With typical illumination systems with 5-20 nm spectral widths, photo-conversion yields of $0.6-0.7$ are reached by constant illumination, which allows easily controlling the molecules for a large number of optogenetic purposes.

\section{REFERENCES}

Andel, F., Lagarias, J. C., and Mathies, R. A. (1996). Resonance Raman analysis of chromophore structure in the lumi-R photoproduct of phytochrome. Bochemistry 35, 15997-16008. doi: 10.1021/bi962175k

Andel, F., Hasson, K. C., Gai, F., Anfinrud, P. A., and Mathies, R. A. (1997). Femtosecond time-resolved spectroscopy of the primary photochemistry of phytochrome. Biospectroscopy 3, 421-433.

Auldridge, M. E., and Forest, K. T. (2011). Bacterial phytochromes: more than meets the light. Crit. Rev. Biochem. Mol. Biol. 46, 67-88. doi: 10.3109/10409238.2010.546389

Auldridge, M. E., Satyshur, K. A., Anstrom, D. M., and Forest, K. T. (2012). Structure-guided engineering enhances a phytochrome-based infrared fluorescent protein. J. Biol. Chem. 287, 7000-7009. doi: 10.1074/jbc.M111.295121

Barth, A., and Zscherp, C. (2002). What vibrations tell us about proteins. Q. Rev. Biophys. 35, 369-430. doi: 10.1017/S0033583502003815

Bhattacharya, S., Auldridge, M. E., Lehtivuori, H., Ihalainen, J. A., and Forest, K. T. (2014). Origins of fluorescence in evolved bacteriophytochromes. J. Biol. Chem. 289, 32144-32152. doi: 10.1074/jbc.M114.589739

Borucki, B., von Stetten, D., Seibeck, S., Lamparter, T., Michael, N., Mroginski, M. A., et al. (2005). Light-induced proton release of phytochrome is coupled to the transient deprotonation of the tetrapyrrole chromophore. J. Biol. Chem. 280, 34358-34364. doi: 10.1074/jbc.M505493200

Burgie, E. S., Bussell, A. N., Walker, J. M., Dubiel, K., and Vierstra, R. D. (2014a). Crystal structure of the photosensing module from a red/far-red lightabsorbing plant phytochrome. Proc. Natl. Acad. Sci. U.S.A. 111, 10176-10184. doi: $10.1073 /$ pnas.1403096111

Burgie, E. S., and Vierstra, R. D. (2014). Phytochromes: an atomic perspective on photoactivation and signaling. Plant Cell 26, 4568-4583. doi: 10.1105/tpc.114.131623

Burgie, E. S., Wang, T., Bussell, A. N., Walker, J. M., Li, H., and Vierstra, R. D. (2014b). Crystallographic and electron microscopic analyses of a bacterial phytochrome reveal local and global rearrangements during photoconversion. J. Biol. Chem. 289, 24573-24587. doi: 10.1074/jbc.M114. 571661

Dasgupta, J., Frontiera, R. R., Taylor, K. C., Lagarias, J. C., and Mathies, R. A. (2009). Ultrafast excited-state isomerization in phytochrome revealed by femtosecond stimulated Raman spectroscopy. Proc. Natl. Acad. Sci. U.S.A. 106, 1784-1789. doi: $10.1073 /$ pnas.0812056106

Eilfeld, P., and Rüdiger, W. (1985). Absoprtion spectra of phytochrome intermediates. $Z$ Naturforsch C 40, 109-114.

Essen, L. O., Mailliet, J., and Hughes, J. (2008). The structure of a complete phytochrome sensory module in the Pr ground state. Proc. Natl. Acad. Sci. U.S.A. 105, 14709-14714. doi: 10.1073/pnas.0806477105

Filonov, G. S., Piatkevich, K. D., Ting, L. M., Zhang, J., Kim, K., and Verkhusha, V. V. (2011). Bright and stable near-infrared fluorescent protein for in vivo imaging. Nat. Biotechnol. 29, 757-761. doi: 10.1038/nbt.1918

Fischer, A. J., and Lagarias, J. G. (2004). Harnessing phytochrome's glowing potential. Proc. Natl. Acad. Sci. U.S.A. 101, 17334-17339. doi: 10.1073/pnas.040 7645101

Foerstendorf, H., Benda, C., Gärtner, W., Storf, M., Scheer, H., and Siebert, F. (2001). FTIR studies of phytochrome photoreactions reveal the $\mathrm{C}=\mathrm{O}$ bands of

\section{FUNDING}

Finnish Cultural foundation (for JI and HT, 0131067) and Academy of Finland (for HT and HL, 285461 and 277194, respectively) are acknowledged.

\section{ACKNOWLEDGMENTS}

We thank G. Groenhof, S. Westenhoff, K. T. Forest, and T. Lamparter for insightful discussions of the topic.

the chromophore: consequences for its protonation states, conformation, and protein interaction. Biochemistry 40, 14952-14959. doi: 10.1021/bi0156916

Gasser, C., Taiber, S., Yeh, C.-M., Wittig, C. H., Hegemann, P., Ryu, S., et al. (2014). Engineering of red-light-activated human cAMP/cGMPspecific phosphodiesterase. Proc. Natl. Acad. Sci. U.S.A. 111, 8803-8808. doi: $10.1073 /$ pnas. 1321600111

Heyne, K., Herbst, J., Stehlik, D., Esteban, B., Lamparter, T., Hughes, J., et al. (2002). Ultrafast dynamics of phytochrome from the cyanobacterium synechocystis, reconstituted with phycocyanobilin and phycoerythrobilin. Biophys. J. 82, 1004-1016. doi: 10.1016/S0006-3495(02)75460-X

Inomata, K., Hammam, M. A., Kinoshita, H., Murata, Y., Khawn, H., Noack, S., et al. (2005). Sterically locked synthetic bilin derivatives ad phytochrome Agp1 from Agrobacterium tumenfacies form photosensitive Pr- and Pfr-like adducts. J. Biol. Chem. 280, 24491-24497. doi: 10.1074/jbc.M504710200

Kim, P. W., Rockwell, N. C., Freer, L. H., Chang, C.-W., Martin, S. S., Lagarias, J. C., et al. (2013). Unraveling the primary isomerization dynamics in Cyanobacterial Phytochrome Cph1 with multipulse manipulations. J. Phys. Chem. Lett. 4, 2605-2609. doi: 10.1021/jz401443q

Kim, P. W., Rockwell, N. C., Martin, S. S., Lagarias, J. C., and Larsen, D. S. (2014). Dynamic inhomogeneity in the Photodynamics of Cyanobacterial Phytochrome Cph1. Biochemistry 53, 2818-2826. doi: 10.1021/bi50 $0108 \mathrm{~s}$

Lagarias, J. C., and Rapoport, H. (1980). Chromopeptides from phytochrome. the structure and linkage of the Pr form of the phytochrome chromophore. J. Am. Chem. Soc. 102, 4821-4828. doi: 10.1021/ja00534a042

Lehtivuori, H., Rissanen, I., Takala, H., Bamford, J., Tkachenko, N. V., and Ihalainen, J. A. (2013). Fluorescence properties of the chromophore-binding domain of bacteriophytochrome from Deinococcus radiodurans. J. Phys. Chem. B 117, 11049-11057. doi: 10.1021/jp312061b

Linke, M., Yang, Y., Zienicke, B., Hammam, M. A. S., von Haimberger, T., Zacarias, A., et al. (2013). Electronic transitions and heterogeneity of the bacteriophytochrome Pr absorption band: an angle balanced polarization resolved femtosecond VIS pump-IR probe study. Biophys. J. 105, 1756-1766. doi: 10.1016/j.bpj.2013.08.041

Mathes, T., Ravensbergen, J., Kloz, M., Gleichemann, T., Gallagher, K. D., Woitowich, N. C., et al. (2015). Femto- to microsecond photodynamics of an unusual bacteriophytochrome. J. Phys. Chem. Lett. 6, 239-243. doi: $10.1021 /$ jz502408n

Matysik, J., Hildebrandt, P., Schlamann, W., Braslavsky, S. E., and Schaffner, K. (1995). Fourier-transform resonance raman spectroscopy of intermediates of the phytochrome photocycle. Biochemistry 34, 10497-10507. doi: 10.1021/bi00033a023

Miller, A. E., Fischer, A. J., Laurence, T., Hollars, C. W., Saykally, R. J., Lagarias, J. C., et al. (2006). Single-molecule dynamics of phytochrome-bound fluorophores probed by fluorescence correlation spectroscopy. Proc. Natl. Acad. Sci. U.S.A. 103, 11136-11141. doi: 10.1073/pnas.0604724103

Möglich, A., and Moffat, K. (2010). Engineered photoreceptors as novel optogenetic tools. Photocehm. Photobiol. Sci. 9, 1286-1300. doi: 10.1039/c0pp00167h

Müller, M. G., Lindner, I., Martin, I., Gärtner, W., and Holzwarth, A. R. (2008). Femtosecond kinetics of photoconversion of the highre plant photoreceptor phytochrome carrying native and modified chromophores. Biophys. J. 94, 4370-4382. doi: 10.1529/biophysj.106.091652 
Nieder, J. B., Brecht, M., and Bittl, R. (2009). Dynamic intracomplex heterogeneity of phytochrome. J. Am. Chem. Soc. 131, 69-71. doi: 10.1021/ja8058292

Nieder, J. B., Stojkovic, E. A., Moffat, K., Forest, K. T., Lamparter, T., Bittl, R., et al. (2013). Pigment-protein interactions in phytochromes probed by fluorescence line narrowing spectroscopy. J. Phys. Chem. B 117, 14940 - 14950. doi: $10.1021 / \mathrm{jp} 409110 \mathrm{q}$

Otto, H., Lamparter, T., Borucki, B., Hughes, J., and Heyn, M. P. (2003). Dimerization and inter-chromophore distance of Cph1 phytochrome from synechocystis, as monitored by fluorescence homo and hetero energy transfer. Biochemistry 42, 5885-5895. doi: 10.1021/bi026946y

Piatkevich, K. D., Subach, F. V., and Verkhusha, V. V. (2013a). Far-red light photoactivable near-infrared fluorescent proteins engineered from a bacterial phytochrome. Nat. Commun. 4, 1-10. doi: 10.1038/ncomms3153

Piatkevich, K. D., Subach, F. V., and Verkhusha, V. V. (2013b). Engineering of bacterial phytochromes for near-infrared imaging, sensing, and lightcontrol in mammals. Chem. Soc. Rev. 42, 3441-3452. doi: 10.1039/ c3cs35458j

Piworski, P., Ritter, E., Hofmann, K.-P., Hildebrandt, P., von Stetten, D., Scheerer, P., et al. (2010). Light-induced activation of bacterial phytochrome agp 1 monitored by static and time-resolved FTIR spectroscopy. Chemphyschem 11, 1207 - 1214. doi: $10.1002 / \mathrm{cphc} .200901008$

Rockwell, N. C., Shang, L., Martin, S. S., and Lagarias, J. C. (2009). Distinct classes of red/far-red photochemistry within the phytochrome superfamily. Proc. Natl. Acad. Sci. U.S.A. 106, 6123-6127. doi: 10.1073/pnas.0902370106

Rockwell, N. C., Su, Y. S., and Lagarias, J. C. (2006). Phytochrome structure and signaling mechanisms. Annu. Rev. Plant Biol. 57, 837-858 doi: 10.1146/annurev.arplant.56.032604.144208

Schumann, C., Groß, R., Michael, N., Lamparter, T., and Diller, R. (2007). Sub-picosecond mid-infrared spectroscopy of phytochrome Agp1 from agrobacterium tumefaciens. Chemphyschem 8, 1657-1663. doi: 10.1002/cphc.200700210

Schwinté, P., Gärtner, W., Sharda, S., Mroginski, M.-A., Hildebrandt, P., and Siebert, F. (2009). The photoreactions of recombinant phytochrome CphA from the cyanobacterium Calothrix PCC7601: a low-temperature UV-Vis and FTIR study. Photochem. Photobiol. 85, 239-249. doi: 10.1111/j.17511097.2008.00426.x

Shcherbakova, D. M., Baloban, M., and Verkhusha, V. V. (2015). Near-infrared fluorescent proteins engineered from bacterial phytochromes. Curr. Opin. Chem. Biol. 27, 52-63. doi: 10.1016/j.cbpa.2015.06.005

Shcherbakova, D. M., and Verkhusha, V. V. (2013). Near-infrared fluorescent proteins for multicolor in vivo imaging. Nat. Methods 10,751-754. doi: 10.1038/ nmeth. 2521

Shimizu-Sato, S., Huq, E., Tepperman, J. M., and Quail, P. H. (2002). A lightswitchable gene promoter system. Nat. Biotechnol. 20, 1041-1044. doi: 10.1038/ nbt734

Shu, X., Royant, A., Lin, M. Z., Aguilera, T. A., Lev-Ram, V., Steinbach, P. A., et al. (2009). Mammalian expression of infrared fluorescent proteins engineered from a bacterial phytochrome. Science 324, 804-807. doi: $10.1126 /$ science. 1168683

Sineshchekov, V. (1995). Photobiophysics and photobiochemistry of the heterogenous phytochrome system. Biochim. Biophys. Acta 1228, 125-164. doi: 10.1016/0005-2728(94)00173-3

Sineshchekov, V., Mailliet, J., Psakis, G., Feilke, K., Kopycki, J., Zeidler, M., et al. (2014). Tyrosine 263 in cyanobacterial phytochrome Cph1 optimizes photochemistry at the prelumi-R $\longrightarrow$ lumi-R step. Photochem. Photobiol. 90, 786-795. doi: 10.1111/php. 12263

Song, C., Psakis, G., Lang, C., Mailliet, J., Gärtner, W., Hughes, J., et al. (2011). Two ground state isoforms and a chromophore D-ring photoflip triggering extensive intramolecular changes in a canonical phytochrome. Proc. Natl. Acad. Sci. U.S.A. 108, 3842-3847. doi: 10.1073/pnas. 1013377108

Spillane, K. M., Dasgupta, J., Lagarias, J. C., and Mathies, R. A. (2009). Homogeneity of Phytochrome $\mathrm{Cph} 1$ vibronic absorption revealed by resonance raman intensity analysis. J. Am. Chem. Soc. 131, 13946-13948. doi: 10.1021/ ja905822m

Stojkovic, E. A., Toh, K. C., Alexandre, M. T. A., Baclayon, M., Moffat, K., and Kennis, J. T. M. (2015). FTIR spectroscopy revealing light-dependent refolding of the conserved tongue region of bacteriophytochrome. J. Phys. Chem. Lett. 5, 2512-2515. doi: 10.1021/jz501189t
Striker, G., Subramaniam, V., Seidel, C. A. M., and Volkmer, A. (1999). Photochromicity and fluorescence lifetimes of green fluorescent protein. J. Phys. Chem. B 40, 8612-8617. doi: 10.1021/jp991425e

Takala, H., Björling, A., Berntsson, O., Lehtivuori, H., Niebling, S., Hoernke, M., et al. (2014a). Signal amplification and transduction in phytochrome photosensors. Nature 509, 245-248. doi: 10.1038/nature13310

Takala, H., Lehtivuori, H., Hammarén, H., Hytönen, V. P., and Ihalainen, J. A. (2014b). Connection between absorption properties and conformational changes in deinococcus radiodurans phytochrome. Biochemistry 53, 7076-7085. doi: 10.1021/bi501180s

Toh, K. C., Stojkovic, E. A., Rupenyan, A. B., van Stokkum, I. H., Salumbides, M., Groot, M. L., et al. (2011b). Primary reactions of bacteriophytochrome observed with ultrafast mid-infrared spectroscopy. J. Phys. Chem. A 115 , 3778-3786. doi: 10.1021/jp106891x

Toh, K. C., Stojkovic, E. A., van Stokkum, I. H., Moffat, K., and Kennis, J. T. (2010). Proton-transfer and hydrogen-bond interactions determine fluorescence quantum yield and photochemical efficiency of bacteriophytochrome. Proc. Natl. Acad. Sci. U.S.A. 107, 9170-9175. doi: 10.1073/pnas.0911535107

Toh, K. C., Stojkovic, E. A., van Stokkum, I. H., Moffat, K., and Kennis, J. T. (2011a). Fluorescence quantum yield and photochemistry of bacteriophytochrome constructs. Phys. Chem. Chem. Phys. 13, 11985-11997. doi: 10.1039/clcp00050k

van Thor, J. J., Boruki, B., Crielaard, W., Otto, H., Lamparter, T., Hughes, J., et al. (2001). Light-induced proton release and proton uptake reactions in the cyanobacterial phytochrome Cph1. Biochemistry 82, 11460 -11471. doi: $10.1021 /$ bi002651d

van Thor, J. J., Ronayne, K. L., and Towrie, M. (2007). Formation of the early photoproduct lumi-R of cyanobacterial phytochrome cph1 observed by ultra-fast mid-infrared spectroscopy. J. Am. Chem. Soc. 129, 126-132. doi: 10.1021/ja0660709

Velazquez Escobar, F., Piwowarski, P., Salewski, J., Michael, N., Fernandez Lopez, M., Rupp, A., et al. (2015). A protonation-coupled feedback mechanism controls the signalling process in bathy phytochromes. Nat. Chem. 7, 423-430. doi: 10.1038/nchem.2225

Vierstra, R. D., and Zhang, J. (2011). Phytochrome signaling: solving the Gordian knot with microbial relatives. Trends Plant Sci. 16, 417-426. doi: 10.1016/j.tplants.2011.05.011

Wagner, J. R., Brunzelle, J. S., Forest, K. T., and Vierstra, R. D. (2005). A lightsensing knot revealed by the structure of the chromophore-binding domain of phytochrome. Nature 438, 325-331. doi: 10.1038/nature04118

Wagner, J. R., Zhang, J., Brunzelle, J. S., Vierstra, R. D., and Forest, K. T. (2007). High resolution structure of Deinococcus bacteriophytochrome yields new insights into phytochrome architecture and evolution. J. Biol. Chem. 282, 12298-12309. doi: 10.1074/jbc.M611824200

Wagner, J. R., Zhang, J., von Stetten, D., Günther, M., Murgida, D. H., Mroginski, M. A., et al. (2008). Mutational analysis of Deinococcus radiodurans bacteriophytochrome reveals key amino acids necessary for the photochromicity and proton exchange cycle of phytochromes. J. Biol. Chem. 283, 12212-12226. doi: 10.1074/jbc.M709355200

Yang, X., Kuk, J., and Moffat, K. (2008). Crystal structure of Pseudomonas aeruginosa bacteriophytochrome: photoconversion and signal transduction. Proc. Natl. Acad. Sci. U.S.A. 105, 14715-14720. doi: 10.1073/pnas.0806718105

Yang, X., Kuk, J., and Moffat, K. (2009). Conformational differences between the Pfr and Pr states in Pseudomonas aeruginosa bacteriophytochrome. Proc. Natl. Acad. Sci. U.S.A. 106, 15639-15644. doi: 10.1073/pnas.0902178106

Yang, X., Ren, Z., Kuk, J., and Moffat, K. (2011). Temperature-scan cryocrystallography reveals reaction intermediates in bacteriophytochrome. Nature 479, 428-432. doi: 10.1038/nature10506

Yang, X., Stojkovic, E. A., and Moffat, K. (2007). Crystal structure of the chromophore binding domain of an unusual bacteriophytochrome, RpBphP3, reveals residues that modulate photoconversion. Proc. Natl. Acad. Sci. U.S.A. 104, 12571-12576. doi: 10.1073/pnas.0701737104

Yang, Y., Linke, M., von Haimberger, T., Hahn, J., Matute, R., Gonzailez, L., et al. (2012). Real-time tracking of phytochrome's orientational changes during Pr photoisomerization. J. Am. Chem. Soc. 134, 1408-1411. doi: 10.1021/ja20 9413d

Yang, Y., Linke, M., von Haimberger, T., Matute, R., Gonzailez, L., Schmieder, P., et al. (2014). Active and silent chromophore isoforms for phytochrome 
Pr photoisomerization: an alternative evolutionary strategy to optimize photoreaction quantum yields. Struct. Dyn. 1, 014701. doi: 10.1063/1.4865233

Yu, D., Gustafson, W. C., Han, C., Lafaye, C., Noirclerc-Savoye, M., Ge, W. P., et al. (2014). An improved monomeric infrared fluorescent protein for neuronal and tumour brain imaging. Nat. Commun. 5, 3626. doi: 10.1038/ ncomms 4626

Zienicke, B., Chen, L.-Y., Khawn, H., Hammam, M. A. S., Kinoshita, H., Reichert, J., et al. (2011). Fluorescence of phytochrome adducts with synthetic locked chromophores. J. Biol. Chem. 286, 1103-1113. doi: 10.1074/jbc.M110. 155143
Conflict of Interest Statement: The authors declare that the research was conducted in the absence of any commercial or financial relationships that could be construed as a potential conflict of interest.

Copyright $\odot 2015$ Ihalainen, Takala and Lehtivuori. This is an open-access article distributed under the terms of the Creative Commons Attribution License (CC BY). The use, distribution or reproduction in other forums is permitted, provided the original author(s) or licensor are credited and that the original publication in this journal is cited, in accordance with accepted academic practice. No use, distribution or reproduction is permitted which does not comply with these terms. 